\title{
PARTIAL CROSSED PRODUCTS AND FULLY WEAKLY PRIME RINGS
}

\author{
WAGNER CORTES AND MARLON SOARES
}

\begin{abstract}
In this paper, we study the necessary and sufficient conditions for the partial crossed product to be a fully weakly prime ring. Moreover, we give a description of the prime radical of the partial crossed product when the base ring is a fully weakly prime ring.
\end{abstract}

1. Introduction. Partial actions of groups have been introduced in the theory of operator algebras as a general approach to studying $C^{*}$ - algebras by partial isometries (see, in particular, $[\mathbf{1 1}, \mathbf{1 2}]$ ), and crossed products classically, which was well established in [8]. They are the center of the rich interplay between dynamical systems and operator algebras (see, for instance, $[\mathbf{1 7}, \mathbf{2 0}]$ ). The general notion of the (continuous) twisted partial action of a locally compact group on a $C^{*}$-algebra and the corresponding crossed product were introduced in [11]. Algebraic counterparts for some notions mentioned above were introduced and studied in [7], stimulating further investigations (see, for instance, $[\mathbf{2}, \mathbf{1 0}, \mathbf{1 3}]$, and the references therein). In particular, twisted partial actions of groups on abstract rings and corresponding crossed products were recently introduced in [8].

In this article, we describe the prime radical of partial crossed products when the base ring is a fully weakly prime ring (see [14] for more details about this theory). We describe the necessary and sufficient conditions for the partial crossed product to be fully weakly prime and, as a consequence of our techniques, we generalize some of the results in [15]. Moreover, we study the necessary and sufficient

2010 AMS Mathematics subject classification. Primary 16D25, 16N60, 16N99, 16S35, 16W22.

Keywords and phrases. Partial crossed product, fully weakly prime rings, prime radical.

The second author was supported by Conselho Nacional de Desenvolvimento Científico e Tecnológico, CNPq, Brazil.

Received by the editors on January 14, 2013, and in revised form on October 15, 2014.

DOI:10.1216/RMJ-2016-46-4-1107

Copyright (C)2016 Rocky Mountain Mathematics Consortium 
conditions for the partial crossed product to be almost fully prime, thus generalizing some of the results in [15].

In Section 2, we provide definitions and results that will be used throughout the article. Moreover, we study matrix rings over fully weakly prime rings.

In Section 3, we describe the prime radical of a partial crossed product when the base ring is a fully weakly prime ring.

In Section 4, we study the necessary and sufficient conditions for the partial crossed product to be a fully weakly prime and, as a consequence of our techniques, we study when the partial crossed product is a fully prime ring. Moreover, we describe the necessary and sufficient conditions for the partial crossed products to be almost fully prime. We also give some examples to show that our results are not an easy generalization of the global case.

2. Preliminaries. In this section, we review the definitions and results that will be used throughout this article. Moreover, we present some new results.

Recall that the multiplier ring of $\mathcal{M}(A)$ of an associative nonnecessarily unital ring $A$ is the set

$$
\mathcal{M}(A)=\left\{(R, L) \in \operatorname{End}\left({ }_{A} A\right) \times \operatorname{End}\left(A_{A}\right) ;(a R) b=a(L b)\right\},
$$

for all $a, b \in A$, with the following operations:

(i) $(\mathcal{R}, L)+\left(\mathcal{R}^{\prime}, L^{\prime}\right)=\left(\mathcal{R}+\mathcal{R}^{\prime}, L+L^{\prime}\right)$;

(ii) $(\mathcal{R}, L)\left(\mathcal{R}^{\prime}, L^{\prime}\right)=\left(\mathcal{R}^{\prime} \circ \mathcal{R}, L \circ L^{\prime}\right)$.

Here, we use the right hand side notation for homomorphisms of left $A$-modules, while the usual notation shall be used for homomorphisms of right modules. In particular, we write

$$
a \longmapsto a \mathcal{R} \quad \text { and } \quad a \longmapsto L a
$$

for $\mathcal{R}:{ }_{A} A \rightarrow{ }_{A} A, L: A_{A} \rightarrow A_{A}$ with $a \in A$. For the multiplier $w=(\mathcal{R}, L) \in \mathcal{M}(A)$ and $a \in A$, we set $a w=a \mathcal{R}$ and $w a=L a$. Thus, one always has $(a w) b=a(w b)$, for all $a, b \in A$. The first (respectively, second) components of the elements of $\mathcal{M}(A)$ are called right (respectively, left) multipliers of $A$. It is convenient to point out that, if $A$ is a unital ring, then we have that $A \simeq \mathcal{M}(A)$ (see [7, 
Proposition 2.3]). So, in this case, each invertible multiplier may be considered to be an invertible element of $A$.

Definition 2.1. [9, Definition 2.1]. A twisted partial action of a group $G$ on a $\operatorname{ring} R$ is a triple,

$$
\alpha=\left(\left\{D_{g}\right\}_{g \in G},\left\{\alpha_{g}\right\}_{g \in G},\left\{w_{g, h}\right\}_{(g, h) \in G \times G}\right),
$$

where, for each $g \in G, D_{g}$ is a two-sided ideal of $R$ and $\alpha_{g}: D_{g^{-1}} \rightarrow D_{g}$ is an isomorphism of rings. For each $(g, h) \in G \times G, w_{g, h}$ is an invertible element from $\mathcal{M}\left(D_{g} D_{g h}\right)$, satisfying the following postulates, for all $g, h, t \in G$ :

(i) $D_{g}^{2}=D_{g}$ and $D_{g} D_{h}=D_{h} D_{g}$;

(ii) $D_{e}=R$ and $\alpha_{e}$ is the identity map of $R$;

(iii) $\alpha_{g}\left(D_{g^{-1}} D_{h}\right)=D_{g} D_{g h}$;

(iv) $\alpha_{g} \circ \alpha_{h}(a)=w_{g, h} \alpha_{g h}(a) w_{g, h}^{-1}$, for all $a \in D_{h^{-1}} D_{h^{-1} g^{-1}}$;

(v) $w_{g, e}=w_{e, g}=1$;

(vi) $\alpha_{g}\left(a w_{h, t}\right) w_{g, h t}=\alpha_{g}(a) w_{g, h} w_{g h, t}$, for all $a \in D_{g^{-1}} D_{h} D_{h t}$.

Note that, if $w_{g, h}=1_{\mathcal{M}\left(D_{g} D_{g h}\right)}$, for all $g, h \in G$, then we have the partial action defined by Dokuchaev and Exel [7, Definition 1.1]. When $D_{g}=R$, for all $g \in G$, we have that $\alpha$ is a twisted global action.

Let $\beta=\left(T,\left\{\beta_{g}\right\}_{g \in G},\left\{u_{g, h}\right\}_{(g, h) \in G \times G}\right)$ be a twisted global action of a group $G$ on a (non-necessarily unital) ring $T$, and let $R$ be an ideal of $T$ generated by a central idempotent $1_{R}$. We can restrict $\beta$ for $R$ as follows. Putting $D_{g}=R \cap \beta_{g}(R)=R \beta_{g}(R)$, we have that each $D_{g}$ has identity $1_{R} \beta_{g}\left(1_{R}\right)$. Then, defining $\alpha_{g}=\left.\beta_{g}\right|_{D_{g^{-1}}}$, $g \in G$, Definition 2.1 (i)-(iii) are satisfied. Furthermore, defining $w_{g, h}=u_{g, h} 1_{R} \beta_{g}\left(1_{R}\right) \beta_{g h}\left(1_{R}\right), g, h \in G$, we have that (iv)-(vi) are also satisfied. Thus, we have obtained a twisted partial action of $G$ on $R$.

Definition 2.2. [9, Definition 2.2]. A twisted global action,

$$
\left(T,\left\{\beta_{g}\right\}_{g \in G},\left\{u_{g, h}\right\}_{(g, h) \in G \times G)}\right)
$$

of a group $G$ on an associative (non-necessarily unital) ring $T$ is said to be an enveloping action (or a globalization) for a twisted partial 
action $\alpha$ of $G$ on a ring $R$, if there exists a ring monomorphism $\varphi: R \rightarrow T$ such that, for all $g$ and $h$ in $G$ :

(i) $\varphi(R)$ is an ideal of $T$;

(ii) $T=\sum_{g \in G} \beta_{g}(\varphi(R))$;

(iii) $\varphi\left(D_{g}\right)=\varphi(R) \cap \beta_{g}(\varphi(R))$;

(iv) $\varphi \circ \alpha_{g}(a)=\beta_{g} \circ \varphi(a)$, for all $a \in D_{g^{-1}}$;

(v) $\varphi\left(a w_{g, h}\right)=\varphi(a) u_{g, h}$ and $\varphi\left(w_{g, h} a\right)=u_{g, h} \varphi(a)$, for all $a \in D_{g} D_{g h}$.

In [9, Theorem 4.1], the authors studied the necessary and sufficient conditions for a twisted partial action $\alpha$ of a group $G$ on a ring $R$ which has an enveloping action. Moreover, they studied which rings satisfy such conditions.

Suppose that $(R, \alpha, w)$ has an enveloping action $(T, \beta, u)$. In this case, we may assume that $R$ is an ideal of $T$, and we can rewrite the conditions of Definition (2.2) as follows:

(i') $R$ is an ideal of $T$;

(ii') $T=\sum_{g \in G} \beta_{g}(R)$;

(iii') $D_{g}=R \cap \beta_{g}(R)$, for all $g \in G$;

(iv') $\alpha_{g}(a)=\beta_{g}(a)$, for all $a \in D_{g^{-1}}$ and $g \in G$;

$\left(\mathrm{v}^{\prime}\right) a w_{g, h}=a u_{g, h}$ and $w_{g, h} a=u_{g, h} a$, for all $a \in D_{g} D_{g h}$ and $g, h \in G$.

Given a twisted partial action $\alpha$ of a group $G$ on a ring $R$, we recall from [8, Definition 2.2] that the partial crossed product $R *_{\alpha}^{w} G$ is the direct sum,

$$
\bigoplus_{g \in G} D_{g} \delta_{g}
$$

where $\delta_{g}$ 's are symbols, with the usual addition and multiplication defined by the rule,

$$
\left(a_{g} \delta_{g}\right)\left(b_{h} \delta_{h}\right)=\alpha_{g}\left(\alpha_{g}^{-1}\left(a_{g}\right) b_{h}\right) w_{g, h} \delta_{g h} .
$$

By [8, Theorem 2.4], we have that $R *_{\alpha}^{w} G$ is an associative ring whose identity is $1_{R} \delta_{1}$. Moreover, we have the injective morphism $\phi: R \rightarrow R *_{\alpha}^{w} G$, defined by $r \mapsto r \delta_{1}$, and we can consider $R *_{\alpha}^{w} G$ an extension of $R$. 
From now on, we assume that

$$
\alpha=\left(\left\{D_{g}\right\}_{g \in G},\left\{\alpha_{g}\right\}_{g \in G},\left\{w_{g, h}\right\}_{(g, h) \in G \times G}\right)
$$

is a twisted partial action of a group $G$ on a $\operatorname{ring} R$ such that all the ideals $D_{g}, g \in G$, are generated by central idempotents $1_{g}$. Note that this is not sufficient for a twisted partial action of a group $G$ on $R$ to have an enveloping action (see [11, Theorem 4.1]).

Definition 2.3. Let $\alpha$ be a twisted partial action of a group $G$ on a ring $R$. An ideal $I$ of $R$ is said to be $\alpha$-invariant if $\alpha_{g}\left(I \cap D_{g^{-1}}\right) \subseteq I \cap D_{g}$, for all $g \in G$.

Note that the definition above is equivalent to $\alpha_{g}\left(I \cap D_{g^{-1}}\right)=I \cap D_{g}$, for all $g \in G$. If $I$ is an $\alpha$-invariant ideal of $R$, then we define $I *{ }_{\alpha}^{w} G$ as the set of all finite sums

$$
\sum_{g \in G} a_{g} \delta_{g},
$$

such that $a_{g} \in I \cap D_{g}$, for all $g \in G$, with the usual addition and multiplication determined by the rule,

$$
\left(a_{g} \delta_{g}\right)\left(b_{h} \delta_{h}\right)=a_{g} \alpha_{g}\left(b_{h} 1_{g^{-1}}\right) w_{g, h} \delta_{g h} .
$$

The proof of the next lemma follows the same methods of $[\mathbf{1 8}$, page 132]. Thus, we omit the proof here.

Lemma 2.4. Let $\alpha$ be a twisted partial action of a group $G$ on $R$.

(i) If $A$ is an ideal of $R *_{\alpha}^{w} G$, then $A \cap R$ is an $\alpha$-invariant ideal of $R$ such that $(A \cap R) *_{\alpha}^{w} G \subseteq A$.

(ii) If $I$ is an $\alpha$-invariant ideal of $R$, then $I *{ }_{\alpha}^{w} G$ is an ideal of $R *_{\alpha}^{w} G$ such that $\left(I *_{\alpha}^{w} G\right) \cap R=I$.

Definition 2.5. Let $\alpha$ be a twisted partial action of a group $G$ on $R$.

(i) An $\alpha$-invariant ideal $J$ of $R$ is said to be $\alpha$-semiprime if, for an $\alpha$-invariant ideal $K$ of $R, K^{2} \subseteq J$ implies that $K \subseteq J$. Moreover, $R$ is said to be $\alpha$-semiprime if the ideal (0) is $\alpha$-semiprime.

(ii) An $\alpha$-invariant ideal $P$ of $R$ is said to be $\alpha$-prime if, for any $\alpha$ invariant ideals $I$ and $J$ of $R$ with $I J \subseteq P$, we have that either 
$I \subseteq P$ or $J \subseteq P$. Moreover, $R$ is said to be $\alpha$-prime if the ideal (0) is $\alpha$-prime.

(iii) The $\alpha$-prime radical of $R$ is the intersection of all $\alpha$-prime ideals of $R$, and we denote it by $\operatorname{Nil}_{\alpha}(R)$.

The proof of Lemma 2.6 is similar to [18, Lemma 14.1], and we write it here for the reader's convenience.

Lemma 2.6. Let $\alpha$ be a twisted partial action of a group $G$ on $R$.

(i) If $Q$ is a prime ideal of $R *{ }_{\alpha}^{w} G$, then $Q \cap R$ is an $\alpha$-prime ideal of $R$.

(ii) If $P$ is an $\alpha$-prime ideal of $R$, then there exists a prime ideal $Q$ of $R *_{\alpha}^{w} G$ such that $Q \cap R=P$.

Proof.

(i) Let $Q$ be a prime ideal of $R *_{\alpha}^{w} G$, and let $I$ and $J$ be $\alpha$-invariant ideals of $R$ such that $I, J \subseteq Q \cap R$. Then, $\left(I *_{\alpha}^{w} G\right)\left(J *_{\alpha}^{w} G\right) \subseteq Q$. By the fact that $Q$ is prime, we have that either $I *_{\alpha}^{w} G \subseteq Q$ or $J *_{\alpha}^{w} G \subseteq Q$. Thus, either $I \subseteq Q \cap R$ or $J \subseteq Q \cap R$. Hence, $Q \cap R$ is an $\alpha$-prime ideal of $R$.

(ii) Let $P$ be an $\alpha$-prime ideal of $R$. Then, by Lemma 2.4 (ii), we have that $\left(P *_{\alpha}^{w} G\right) \cap R=P$. By Zorn's lemma, there exists an ideal $Q$ in $R *{ }_{\alpha}^{w} G$, maximal with the property $Q \cap R=P$. Now, it is easy to see that $Q$ is a prime ideal of $R *{ }_{\alpha}^{w} G$ such that $Q \cap R=P$.

Lemma 2.7. Let $\alpha$ be a twisted partial action of a group $G$ on $R$, and let $(T, \beta, u)$ be its enveloping action. If

$$
M=\left\{\sum_{g \in G} a_{g} \delta_{g}: a_{g} \in R\right\}
$$

and

$$
N=\left\{\sum_{g \in G} a_{g} \delta_{g}: a_{g} \in \beta_{g}(R)\right\},
$$

then the following conditions hold:

(i) $M\left(T *_{\beta}^{u} G\right) \subseteq M$; 
(ii) $\left(T *_{\beta}^{u} G\right) N \subseteq N$;

(iii) $\left(R *_{\alpha}^{w} G\right) M \subseteq M$;

(iv) $N\left(R *_{\alpha}^{w} G\right) \subseteq N$;

(v) $M N=R *_{\alpha}^{w} G$;

(vi) $N M=T *_{\beta}^{u} G$;

(vii) $M\left(R *_{\alpha}^{w} G\right) \subseteq R *_{\alpha}^{w} G$;

(viii) $\left(R *_{\alpha}^{w} G\right) N \subseteq R *_{\alpha}^{w} G$.

Proof. The proofs of (i)-(vi) are similar to those presented in [7, Propositions 5.1-5.3].

The proof of (vii) is given by the fact that $M M \subseteq M$ and, using (v), we have that $M R *_{\alpha}^{w} G=M M N \subseteq M N=R *_{\alpha}^{w} G$.

To prove (viii), note that $N N \subseteq N$, and using (v), we obtain the result.

Recall from [6, page 345] that a ring $S$ is a left (right) s-unital if, for any $r \in S$, we have that $r \in S r(r \in r S)$. A ring $S$ is said to be $s$-unital if it is right and left $s$-unital. We clearly have that every unital ring is $s$-unital. Note that, if $R$ is $s$-unital and $(T, \beta, u)$ is the enveloping action of $(R, \alpha, w)$, then by [6, Remark 2.5], we have that $T$ is $s$-unital. Using this fact, we obtain Lemma 2.8 which appears in the proof of [9, Theorem 3.1].

Lemma 2.8. Let $R$ be an s-unital ring, and let $\alpha$ be a twisted partial action of a group $G$ on $R$ with enveloping action $(T, \beta, u)$. Then, $T *{ }_{\beta}^{u} G$ is s-unital.

It is convenient to point out that $R$ being unital implies that $T$ and $T *_{\beta}^{u} G$ are $s$-unital. This implication will be used throughout the paper without further reference.

The proof of the following lemma is standard, and we omit the proof here.

Lemma 2.9. Let $P^{\prime}$ be an ideal of $T *_{\beta}^{u} G$. Then, $P^{\prime} \cap\left(R *_{\alpha}^{w} G\right)$ is an ideal of $R *_{\alpha}^{w} G$. 
Lemma 2.10. There exists a bijective correspondence, via contraction, between the set of the ideals of $R *_{\alpha}^{w} G$ and the set of the ideals of $T *_{\beta}^{u} G$.

Proof. Let $P$ be an ideal of $R *_{\alpha}^{w} G$. Clearly, NPM is a subring of $T *_{\beta}^{u} G$. Since $M\left(T *_{\beta}^{u} G\right) \subseteq M$ and $\left(T *_{\beta}^{u} G\right) N \subseteq N$, it follows that

$$
N P M\left(T *_{\beta}^{u} G\right) \subseteq N P M
$$

and

$$
\left(T *_{\beta}^{u} G\right) N P M \subseteq N P M .
$$

Thus, $N P M$ is an ideal of $T *_{\beta}^{u} G$.

Since $P=1_{R} P 1_{R}, 1_{R} \in N$ and $1_{R} \in M$, then $P \subseteq N P M \cap\left(R *_{\alpha}^{w} G\right)$. Now, for each $x \in N P M \cap\left(R *_{\alpha}^{w} G\right)$, we have that $x=1_{R} x 1_{R} \in$ $1_{R} N P M 1_{R}$. By the fact that $M N=R *_{\alpha}^{w} G$, we have that

$$
x=1_{R} x 1_{R} \in 1_{R} N P M 1_{R} \subseteq\left(R *_{\alpha}^{w} G\right) P\left(R *_{\alpha}^{w} G\right) \subseteq P .
$$

Thus, $N P M \cap\left(R *_{\alpha}^{w} G\right) \subseteq P$, and it follows that $N P M \cap\left(R *_{\alpha}^{w} G\right)=P$.

Next, let $P^{\prime}$ be an ideal of $T *_{\beta}^{u} G$. Since $M\left(T *_{\beta}^{u} G\right) \subseteq M$ and $M N=R *_{\alpha}^{w} G$, we obtain that $M P^{\prime} N \subseteq R *_{\alpha}^{w} G$, and we easily see that $M P^{\prime} N$ is a subring of $R *_{\alpha}^{w} G$. By the fact that $\left(R *_{\alpha}^{w} G\right) M \subseteq M$ and $N\left(R *_{\alpha}^{w} G\right) \subseteq N$, it follows that

$$
\left(R *_{\alpha}^{w} G\right) M P^{\prime} N \subseteq M P^{\prime} N
$$

and

$$
M P^{\prime} N\left(R *_{\alpha}^{w} G\right) \subseteq M P^{\prime} N .
$$

Thus, $M P^{\prime} N$ is an ideal of $R *_{\alpha}^{w} G$.

By the fact that $M P^{\prime} N \subseteq R *_{\alpha}^{w} G$ and $P^{\prime}$ is an ideal of $T *_{\beta}^{u} G$, we have that $M P^{\prime} N \subseteq P^{\prime} \cap\left(R *_{\alpha}^{w} G\right)$. Now, for each $x \in P^{\prime} \cap\left(R *_{\alpha}^{w} G\right)$, we have

$$
x=1_{R} x 1_{R} \in 1_{R} P^{\prime} 1_{R} \subseteq M P^{\prime} N .
$$

Thus, $P^{\prime} \cap\left(R *_{\alpha}^{w} G\right) \subseteq M P^{\prime} N$, and it follows that $P^{\prime} \cap\left(R *_{\alpha}^{w} G\right)=$ $M P^{\prime} N$.

The next corollary is a direct consequence of the proof of Lemma 2.10. 
Corollary 2.11. Let $P^{\prime}$ be an ideal of $T *_{\beta}^{u} G$, and let $P$ be an ideal of $R *{ }_{\alpha}^{w} G$ such that $P=P^{\prime} \cap\left(R *{ }_{\alpha}^{w} G\right)$. Then $P=M P^{\prime} N$ and $P^{\prime}=N P M$.

Recall that, given two rings $R$ and $S$, bimodules ${ }_{R} U_{S}$ and ${ }_{S} V_{R}$ and the maps,

$$
\theta: U \otimes_{S} V \longrightarrow R \text { and } \psi: V \otimes_{R} U \longrightarrow S
$$

the collection $(R, S, U, V, \theta, \psi)$ is said to be a Morita context if the set,

$$
\left(\begin{array}{ll}
R & U \\
V & S
\end{array}\right)
$$

with the usual formal operations of $2 \times 2$ matrices, is a ring. As in Lemma 2.7, consider

$$
M=\left\{\sum_{g \in G} a_{g} \delta_{g}: a_{g} \in R\right\}
$$

and

$$
N=\left\{\sum_{g \in G} a_{g} \delta_{g}: a_{g} \in \beta_{g}(R)\right\} .
$$

Using Lemma 2.7 and arguments similar to those presented in the proof of [9, Theorem 3.1], we have the Morita context

$$
\left(R *_{\alpha}^{w} G, T *_{\beta}^{u} G, M, N, \theta, \psi\right),
$$

where $\theta$ and $\psi$ are the obvious maps.

The next result is a generalization of [13, Proposition 5.1].

Lemma 2.12. There exists a bijective correspondence, via contraction, between the set of the prime ideals of $R *_{\alpha}^{w} G$ and the set of the prime ideals of $T *_{\beta}^{u} G$.

Proof. Let $P^{\prime}$ be a prime ideal of $T *_{\beta}^{u} G$, and assume that $I$ and $J$ are ideals of $R *{ }_{\alpha}^{w} G$ such that

$$
I J \subseteq P^{\prime} \cap\left(R *_{\alpha}^{w} G\right)=P .
$$


Since $I J \subseteq P$, it follows that

$$
I^{\prime} J^{\prime}=(N I M)(N J M) \subseteq N I J M \subseteq N P M=P^{\prime},
$$

so either $I^{\prime} \subseteq P^{\prime}$ or $J^{\prime} \subseteq P^{\prime}$. Thus, either

$$
I=M I^{\prime} N \subseteq M P^{\prime} N=P
$$

or

$$
J=M J^{\prime} N \subseteq M P^{\prime} N=P .
$$

Hence,

$$
P=P^{\prime} \cap\left(R *_{\alpha}^{w} G\right)
$$

is a prime ideal of $R *_{\alpha}^{w} G$. Analogously, it can be seen that, if $P$ is a prime ideal of $R *_{\alpha}^{w} G$, then there exists a prime ideal $P^{\prime}$ of $T *_{\beta}^{u} G$ such that $P=P^{\prime} \cap\left(R *_{\alpha}^{w} G\right)$.

In what follows, we will see some consequences of the last result that will be used throughout the rest of the article.

Corollary 2.13. There exists a bijective correspondence, via contraction, between the set of the prime ideals $P^{\prime}$ of $T *{ }_{\beta}^{u} G$ such that $P^{\prime} \cap T=0$ and the set of the prime ideals $P$ of $R *_{\alpha}^{w} G$ such that $P \cap R=0$.

Proof. Let $P^{\prime}$ be a prime ideal of $T *_{\beta}^{u} G$ such that $P^{\prime} \cap T=0$. Then, by Lemma 2.12 , there exists a prime ideal $P$ of $R *_{\alpha}^{w} G$ such that $P=P^{\prime} \cap\left(R *_{\alpha}^{w} G\right)$. Thus,

$$
\begin{aligned}
P \cap R & =\left(P^{\prime} \cap\left(R *_{\alpha}^{w} G\right)\right) \cap R \\
& =P^{\prime} \cap\left(\left(R *_{\alpha}^{w} G\right) \cap R\right) \\
& =P^{\prime} \cap R \subseteq P^{\prime} \cap T=0 .
\end{aligned}
$$

Now, let $P$ be a prime ideal of $R *_{\alpha}^{w} G$ such that $P \cap R=0$. Then, by Lemma 2.12 , there exists a prime ideal $P^{\prime}$ of $T *_{\beta}^{u} G$ such that $P=P^{\prime} \cap\left(R *_{\alpha}^{w} G\right)$. Thus,

$$
P^{\prime} \cap R=P^{\prime} \cap\left(\left(R *_{\alpha}^{w} G\right) \cap R\right)=\left(P^{\prime} \cap\left(R *_{\alpha}^{w} G\right)\right) \cap R=P \cap R=0,
$$


and it follows that $\left(P^{\prime} \cap T\right) 1_{R}=0$. Since $P^{\prime} \cap T$ is $\beta$-invariant, we have that $\left(P^{\prime} \cap T\right) \beta_{g}\left(1_{R}\right)=0$, for all $g \in G$. By the fact that

$$
T=\sum_{g \in G} \beta_{g}(R)
$$

we obtain that $\left(P^{\prime} \cap T\right) T=0$ and, since $T$ is $s$-unital, it follows that $P^{\prime} \cap T \subseteq\left(P^{\prime} \cap T\right) T=0$.

Let $\alpha$ be a twisted partial action of a group $G$ on $R$, and let $I$ be an $\alpha$-invariant ideal of $R$. We define

$$
I^{*}=\left\{t \in T: \beta_{g}(t) 1_{R} \in I \text { for all } g \in G\right\} .
$$

The proof of the following lemma is analogous to the proof of [2, Lemma 2.3] and will be omitted.

Lemma 2.14. Suppose that $(R, \alpha, w)$ has an enveloping action $(T, \beta, u)$. If $I$ is an $\alpha$-invariant ideal of $R$, then $I^{*}$ is a $\beta$-invariant ideal of $T$, with $I^{*} \cap R=I$. Moreover, for any $\beta$-invariant ideal $J$ of $T$ with $J \cap R=I$, we have $J \subseteq I^{*}$. In addition, if $I$ is $\alpha$-prime, then $I^{*}$ is $\beta$-prime and, conversely, if $J$ is a $\beta$-prime ideal of $T$, then there exists an $\alpha$-prime ideal $I$ of $R$ such that $I^{*}=J$.

Let $I$ be an $\alpha$-invariant ideal of $R$. Then, we can extend the twisted partial action $\alpha$ of $G$ on $R$ to a twisted partial action $\bar{\alpha}$ of $G$ on $R / I$ as follows.

For each $g \in G$, we define

$$
\bar{\alpha}_{g}:\left(D_{g^{-1}}+I\right) / I \longrightarrow\left(D_{g}+I\right) / I
$$

by

$$
\bar{\alpha}_{g}(a+I)=\alpha_{g}(a)+I,
$$

and, for each $(g, h) \in G \times G$, we extend each $w_{g, h}$ to $R / I$ by $\overline{w_{g, h}}=$ $w_{g, h}+I$.

The next corollary generalizes [18, Lemma 16.6 (iii)].

Corollary 2.15. Suppose that $G$ is a finite group. If $P_{1}$ and $P_{2}$ are prime ideals of $R *{ }_{\alpha}^{w} G$ such that $P_{1} \cap R=P_{2} \cap R$, then $P_{1}=P_{2}$. 
Proof. Let $Q=P_{1} \cap R=P_{2} \cap R$. Then, by Lemma 2.6, $Q$ is an $\alpha$-prime ideal of $R$ and, by Lemma 2.14 , we have that the set

$$
Q^{*}=\left\{t \in T: \beta_{g}(t) 1_{R} \in Q \text { for all } g \in G\right\}
$$

is a $\beta$-prime ideal of $T$ such that $Q^{*} \cap R=Q$. By arguments similar to [13, Proposition 2.10], $(R / Q, \bar{\alpha}, \bar{w})$ has an enveloping action $\left(T / Q^{*}, \bar{\beta}, \bar{u}\right)$. Thus, we may assume that

$$
Q=P_{1} \cap R=P_{2} \cap R=0,
$$

$R$ is $\alpha$-prime and $T$ is $\beta$-prime. By Corollary 2.13, there exist prime ideals $P_{1}^{\prime}$ and $P_{2}^{\prime}$ of $T *_{\beta}^{u} G$, such that $P_{1}^{\prime} \cap T=P_{2}^{\prime} \cap T=0$. Hence, by $\left[\mathbf{1 8}\right.$, Theorem 16.6 (iii)], we have that $P_{1}^{\prime}=P_{2}^{\prime}$, and it follows that $P_{1}=P_{2}$.

Corollary 2.16. Suppose that $R$ is an $\alpha$-prime ring and that $G$ is a finite group. A prime ideal $P$ of $R *_{\alpha}^{w} G$ is minimal if and only if $P \cap R=0$.

Proof. Let $P$ be a minimal prime ideal of $R *_{\alpha}^{w} G$. Then, by Lemma 2.12, there exists a prime ideal $P^{\prime}$ of $T *_{\beta}^{u} G$ such that $P^{\prime} \cap$ $\left(R *_{\alpha}^{w} G\right)=P$. We claim that $P^{\prime}$ is minimal. In fact, let $Q^{\prime}$ be a prime ideal of $T *_{\beta}^{u} G$ such that $0 \neq Q^{\prime} \subseteq P^{\prime}$. Thus,

$$
0 \neq Q=Q^{\prime} \cap\left(R *_{\alpha}^{w} G\right) \subseteq P,
$$

and, by assumption, $Q=P$. Hence, $Q^{\prime}=P^{\prime}$. Since $R$ is $\alpha$-prime, then by Lemma 2.14, we have that $T$ is $\beta$-prime. By [18, Theorem 16.2 (i)], we have that $P^{\prime} \cap T=0$, and, by Corollary 2.13, we obtain that $P \cap R=0$.

Conversely, let $P, Q$ be prime ideals of $R *_{\alpha}^{w} G$ such that $P \cap R=0$ and $0 \neq Q \subseteq P$. Then, $Q \cap R \subseteq P \cap R=0$. Thus, $Q \cap R=P \cap R$ and, by Corollary 2.15, we have that $Q=P$. So, $P$ is a minimal prime ideal in $R *_{\alpha}^{w} G$.

Lemma 2.17. If $I$ is a nonzero $\beta$-invariant ideal of $T$, then $I \cap R \neq 0$.

Proof. Suppose that $I \cap R=0$. Then $I 1_{R}=I \cap R=0$. By the fact that $I$ is $\beta$-invariant, we have that $I \beta_{g}\left(1_{R}\right)=0$, for all $g \in G$, and it 
follows that $I T=0$. Since $T$ is $s$-unital, we have that $I \subseteq I T=0$, which is a contradiction.

Definition 2.18. [1, Definition 1.1], [15, page 86]. Let $S$ be a ring.

(i) $S$ is said to be a fully prime ring (FPR) if every ideal of $S$ is prime.

(ii) $S$ is said to be an almost fully prime ring (AFPR) if every proper ideal of $S$ is prime and $S$ is not a prime ring.

Proposition 2.19. [1, Theorem 1.2]. A ring $S$ is an FPR if and only if the set of all the ideals of $S$ is totally ordered by inclusion and all the ideals of $S$ are idempotent.

At this point, it is convenient to point out that any FPR has only one maximal ideal. The following two results were proved in [21, Theorems 2.1, 2.2].

Lemma 2.20. Let $S$ be a ring whose set of ideals is not totally ordered by inclusion. Then $S$ is an AFPR if and only if:

(i) all ideals of $S$ are idempotent and it has exactly two minimal ideals;

(ii) each minimal ideal of $S$ is contained in all nonzero ideals of $S$ that are not minimal ideal;

(iii) the set of the ideals of $S$ that are not minimal is totally ordered by inclusion.

Lemma 2.21. Let $S$ be a ring whose set of ideals is totally ordered by inclusion. Then $S$ is an AFPR if and only if $S$ has only one minimal ideal and every ideal of $S$ except the minimal one is idempotent.

In what follows, we denote the prime radical of a ring $S$, i.e., the intersection of all prime ideals of $S$, by $\mathrm{Nil}_{*}(S)$. The proof of the next result follows directly from Lemmas 2.20 and 2.21 . 


\section{Lemma 2.22.}

(i) If $S$ is an AFPR whose set of ideals is not totally ordered by inclusion, then $\operatorname{Nil}_{*}(S)=P_{1} \cap P_{2}=0$, where $P_{1}$ and $P_{2}$ are the minimal ideals of $S$.

(ii) If $S$ is an AFPR whose set of ideals is totally ordered by inclusion, then $\mathrm{Nil}_{*}(S)=P_{0}$, where $P_{0}$ is the minimal prime ideal of $S$ that is nilpotent.

Definition 2.23. [14, page 1078, Definition]. Let $S$ be a ring.

(i) A proper ideal $I$ of $S$ is said to be a weakly prime ideal if, for any ideals $J$ and $K$ of $R$ with $0 \neq J, K \subseteq I$, we have that either $J \subseteq I$ or $K \subseteq I$.

(ii) $S$ is said to be a fully weakly prime ring (FWPR) if every proper ideal of $S$ is weakly prime.

We conclude this section with some results of independent interest.

By [16, Theorem 3.1], there is a bijective correspondence between the set of ideals of a ring $S$ and the set of ideals of the ring of matrices $\mathbb{M}_{n}(S)$, i.e., let

$$
\{I: I \text { ideal of } S\}
$$

and

$$
\left\{J: J \text { ideal of } M_{n}(S)\right\}
$$

be the sets. The correspondence is given by:

$$
I \longrightarrow M_{n}(I)
$$

and

$$
J \longrightarrow\left\{s \in S: \text { exists } U=\left[a_{i j}\right] \in J \text { such that } a_{11}=s\right\} .
$$

In the next result, we use this fact without further mention.

Proposition 2.24. $S$ is an FWPR if and only if $\mathbb{M}_{n}(S)$ is an FWPR.

Proof. Suppose that $S$ is an FWPR. Let $J$ be a proper ideal of $\mathbb{M}_{n}(S)$, and assume that $A$ and $B$ are ideals of $\mathbb{M}_{n}(S)$ such that 
$0 \neq A B \subseteq J$. Then there exist ideals $I, K$ and $L$ of $S$ such that

$$
J=\mathbb{M}_{n}(I), \quad A=\mathbb{M}_{n}(K), \quad B=\mathbb{M}_{n}(L) \quad \text { and } \quad 0 \neq K L \subseteq I .
$$

By the fact that $S$ is an FWPR, we have that either $K \subseteq I$ or $L \subseteq I$. Thus, either $A \subseteq J$ or $B \subseteq J$. So, $\mathbb{M}_{n}(S)$ is an FWPR.

Conversely, suppose that $\mathbb{M}_{n}(S)$ is an FWPR . Let $K$ be a proper ideal of $\mathbb{M}_{n}(S)$, and assume that $I$ and $J$ are ideals of $S$ such that $0 \neq I, J \subseteq K$. Then,

$$
0 \neq \mathbb{M}_{n}(I) \mathbb{M}_{n}(J) \subseteq \mathbb{M}_{n}(K) .
$$

By assuming $\mathbb{M}_{n}(S)$, either $\mathbb{M}_{n}(J) \subseteq \mathbb{M}_{n}(K)$ or $\mathbb{M}_{n}(I) \subseteq \mathbb{M}_{n}(K)$. Thus, either $I \subseteq K$ or $J \subseteq K$. So, $S$ is an FWPR .

By similar methods to the those of Proposition 2.24, we obtain the following result which contains the result given in [1, Theorem 2.1].

Proposition 2.25. The following statements hold:

(i) $S$ is an FPR if and only if $\mathbb{M}_{n}(S)$ is an FPR.

(ii) $S$ is an $\mathrm{AFPR}$ if and only if $\mathbb{M}_{n}(S)$ is an $\mathrm{AFPR}$.

3. Prime radical of partial crossed products. In this section, we describe the prime radical of partial crossed products when the base ring is a fully weakly prime ring. To do so, we need the following definition.

Definition 3.1. Let $\alpha$ be a twisted partial action of a group $G$ on $R$. We say that a proper $\alpha$-invariant ideal $Q$ of $R$ is weakly $\alpha$-prime if, for any $\alpha$-invariant ideals $A$ and $B$ of $R$ with $0 \neq A B \subseteq Q$, we have that either $A \subseteq Q$ or $B \subseteq Q$.

The proof of the following lemma is analogous to the proof of $[\mathbf{1 4}$, Proposition 1] and we provide it here for the reader's convenience.

Lemma 3.2. Let $P$ be a weakly $\alpha$-prime ideal of $R$ that is not $\alpha$-prime. Then $P^{2}=0$. 
Proof. By assumption, there exist $\alpha$-invariant ideals $I$ and $J$ of $R$ such that

$$
I \nsubseteq P, \quad J \nsubseteq P \text { and } \quad 0=I J \subseteq P .
$$

If $P^{2} \neq 0$, then

$$
0 \neq P^{2} \subseteq(I+P)(J+P) \subseteq P,
$$

which implies that either $I \subseteq P$ or $J \subseteq P$, which is a contradiction.

Given a nonzero element,

$$
a=\sum_{g \in G} a_{g} \delta_{g}
$$

of $R *{ }_{\alpha}^{w} G$, the support of $a$ is defined by

$$
\operatorname{supp}(a)=\left\{g \in G: a_{g} \neq 0\right\} .
$$

Lemma 3.3. If $R$ is semiprime, then $R *_{\alpha}^{w} G$ is semiprime.

Proof. Let

$$
a=\sum_{g \in G} a_{g} \delta_{g} \in R *_{\alpha}^{w} G
$$

be such that $a\left(R *_{\alpha}^{w} G\right) a=0$. Suppose that $a \neq 0$. Then there exists $s \in \operatorname{supp}(a)$. Note that

$$
1_{s^{-1}} \delta_{s^{-1}} a\left(R *_{\alpha}^{w} G\right) 1_{s^{-1}} \delta_{s^{-1}} a \subseteq 1_{s^{-1}} \delta_{s^{-1}} a\left(R *_{\alpha}^{w} G\right) a=0 .
$$

Thus, $1_{s^{-1}} \delta_{s^{-1}} a R 1_{s^{-1}} \delta_{s^{-1}} a=0$, and it follows that

$$
\alpha_{s^{-1}}\left(a_{s}\right) w_{s^{-1}, s} R \alpha_{s^{-1}}\left(a_{s}\right) w_{s^{-1}, s}=0 .
$$

Consequently, $\alpha_{s^{-1}}\left(a_{s}\right) w_{s^{-1}, s}=0$, since $R$ is semiprime. Hence, $a_{s}=0$ is a contradiction because $s \in \operatorname{supp}(a)$. Thus, $R *_{\alpha}^{w} G$ is semiprime.

Since $\mathrm{Nil}_{*}(R)$ is an $\alpha$-invariant ideal of $R$, the twisted partial action $\alpha$ of $G$ on $R$ induces a twisted partial action of $G$ on $R / \mathrm{Nil}_{*}(R)$. We denote this partial action by $\alpha$.

Proposition 3.4. For any twisted partial action $\alpha$ of a group $G$ on $R$, we have

$$
\operatorname{Nil}_{\alpha}(R) *_{\alpha}^{w} G \subseteq \operatorname{Nil}_{*}\left(R *_{\alpha}^{w} G\right) \subseteq \operatorname{Nil}_{*}(R) *_{\alpha}^{w} G
$$


Proof. For any prime ideal $P$ of $R *{ }_{\alpha}^{w} G$, by Lemma (2.6) (i), we obtain that $Q=P \cap R$ is an $\alpha$-prime ideal of $R$. Since $\operatorname{Nil}_{\alpha}(R) \subseteq Q$, then by Lemma 2.4 (i), we have that

$$
\operatorname{Nil}_{\alpha}(R) *_{\alpha}^{w} G \subseteq Q *_{\alpha}^{w} G=(P \cap R) *_{\alpha}^{w} G \subseteq P .
$$

Hence, $\operatorname{Nil}_{\alpha}(R) *_{\alpha}^{w} G \subseteq \operatorname{Nil}_{*}\left(R *_{\alpha}^{w} G\right)$.

Moreover, it is well known that $\operatorname{Nil}_{*}(R)$ is a semiprime ideal of $R$, and thus, $R / \mathrm{Nil}_{*}(R)$ is a semiprime ring. By Lemma 3.3, we have that $\left(R / \mathrm{Nil}_{*}(R)\right) *_{\alpha}^{w} G$ is semiprime. Since

$$
\left(R / \mathrm{Nil}_{*}(R)\right) *_{\alpha}^{w} G \cong\left(R *_{\alpha}^{w} G\right) /\left(\mathrm{Nil}_{*}(R) *_{\alpha}^{w} G\right),
$$

it follows that

$$
\mathrm{Nil}_{*}\left(R *_{\alpha}^{w} G\right) \subseteq \mathrm{Nil}_{*}(R) *_{\alpha}^{w} G
$$

Proposition 3.5. For any twisted partial action $\alpha$ of a group $G$ on $R$, we have

$$
\operatorname{Nil}_{*}\left(R * *_{\alpha}^{w} G\right) \cap R=\operatorname{Nil}_{\alpha}(R) .
$$

Proof. By Lemma 2.6 (ii), for any $\alpha$-prime ideal $Q$ of $R$, there exists a prime ideal $P$ of $R *_{\alpha}^{w} G$ such that $P \cap R=Q$, and so $\mathrm{Nil}_{*}\left(R *_{\alpha}^{w} G\right) \cap R \subseteq P \cap R=Q$. Hence,

$$
\operatorname{Nil}_{*}\left(R *_{\alpha}^{w} G\right) \cap R \subseteq \operatorname{Nil}_{\alpha}(R) .
$$

By Proposition 3.4, we have that $\operatorname{Nil}_{\alpha}(R) *_{\alpha}^{w} G \subseteq \operatorname{Nil}_{*}\left(R *_{\alpha}^{w} G\right)$, and consequently, $\left(\operatorname{Nil}_{\alpha}(R) *_{\alpha}^{w} G\right) \cap R \subseteq \operatorname{Nil}_{*}\left(R *_{\alpha}^{w} G\right) \cap R$. So, by Lemma 2.4 (ii), we have that

$$
\operatorname{Nil}_{\alpha}(R)=\left(\operatorname{Nil}_{\alpha}(R) *_{\alpha}^{w} G\right) \cap R \subseteq \operatorname{Nil}_{*}\left(R *_{\alpha}^{w} G\right) \cap R .
$$

Lemma 3.6. If $R$ is an $\mathrm{FWPR}$, then $\mathrm{Nil}_{*}(R)=\mathrm{Nil}_{\alpha}(R)$.

Proof. By Proposition 3.4, we have that

$$
\operatorname{Nil}_{\alpha}(R) *_{\alpha}^{w} G \subseteq \operatorname{Nil}_{*}(R) *_{\alpha}^{w} G .
$$

Thus,

$$
\operatorname{Nil}_{\alpha}(R)=\left(\operatorname{Nil}_{\alpha}(R) *_{\alpha}^{w} G\right) \cap R \subseteq\left(\operatorname{Nil}_{*}(R) *_{\alpha}^{w} G\right) \cap R=\operatorname{Nil}_{*}(R) .
$$


By [14, Theorem 1], we have that $\operatorname{Nil}_{*}(R)$ is nilpotent. Then, since $\mathrm{Nil}_{*}(R)$ is $\alpha$-invariant and $\operatorname{Nil}_{\alpha}(R)$ is $\alpha$-semiprime, it follows that $\mathrm{Nil}_{*}(R) \subseteq \operatorname{Nil}_{\alpha}(R)$. So, $\operatorname{Nil}_{*}(R)=\operatorname{Nil}_{\alpha}(R)$.

In [14, Theorem 1], the authors studied the prime radical of rings that are FWPR. From now on, for any ring $S$, we denote the sum of all nilpotent ideals of $S$ by $\operatorname{Nil}(S)$, and we denote the Jacobson radical of $S$ by $J(S)$. Next, we give a description of the prime radical of partial crossed products when the base ring is an FWPR.

Theorem 3.7. If $R$ is an FWPR, then

$$
\operatorname{Nil}_{*}\left(R *_{\alpha}^{w} G\right)=\operatorname{Nil}(R) *_{\alpha}^{w} G=\operatorname{Nil}_{\alpha}(R) *_{\alpha}^{w} G=\operatorname{Nil}_{*}(R) *_{\alpha}^{w} G .
$$

Proof. By $\left[\mathbf{1 4}\right.$, Theorem 1] and Lemma 3.6, we have that $\operatorname{Nil}_{*}(R)=$ $\operatorname{Nil}(R)=\operatorname{Nil}_{\alpha}(R)$. By Proposition 3.4,

$$
\operatorname{Nil}_{\alpha}(R) *_{\alpha}^{w} G \subseteq \operatorname{Nil}_{*}\left(R *_{\alpha}^{w} G\right) \subseteq \operatorname{Nil}_{*}(R) *_{\alpha}^{w} G,
$$

it follows that

$$
\operatorname{Nil}_{*}\left(R *_{\alpha}^{w} G\right)=\operatorname{Nil}(R) *_{\alpha}^{w} G=\operatorname{Nil}_{\alpha}(R) *_{\alpha}^{w} G=\operatorname{Nil}_{*}(R) *_{\alpha}^{w} G .
$$

Using [14, Corollary 2] and Theorem 3.7, we obtain the following result.

Corollary 3.8. If $R$ is a Noetherian FWPR, then

$$
\mathrm{Nil}_{*}\left(R *_{\alpha}^{w} G\right)=J(R) *_{\alpha}^{w} G .
$$

Corollary 3.9. Suppose that $R *_{\alpha}^{w} G$ is an FWPR and $R$ is a Noetherian FWPR. Then,

$$
\operatorname{Nil}_{*}\left(R *_{\alpha}^{w} G\right)=J\left(R *_{\alpha}^{w} G\right)=J(R) *_{\alpha}^{w} G .
$$

Proof. By analogous methods to those of [2, Corollary 3.4], $R *_{\alpha}^{w} G$ is Noetherian. Now, using [14, Corollary 2] and Theorem 3.7, we obtain the result.

Lemma 3.10. If $R *_{\alpha}^{w} G$ is an FWPR, then $\left(\operatorname{Nil}_{\alpha}(R)\right)^{2}=0$. 
Proof. By Proposition 3.5, we have that $\operatorname{Nil}_{*}\left(R *_{\alpha}^{w} G\right) \cap R=\operatorname{Nil}_{\alpha}(R)$, and since $R *_{\alpha}^{w} G$ is an FWPR, then by [14, Theorem 1], we have that $\left(\mathrm{Nil}_{*}\left(R * *_{\alpha}^{w} G\right)\right)^{2}=0$. So,

$$
\left(\operatorname{Nil}_{\alpha}(R)\right)^{2}=\left(\operatorname{Nil}_{*}\left(R *_{\alpha}^{w} G\right) \cap R\right)^{2} \subseteq\left(\operatorname{Nil}_{*}\left(R *_{\alpha}^{w} G\right)\right)^{2}=0 .
$$

Let $\alpha$ be a twisted partial action of $Z$ on $R$, and let $(T, \beta, u)$ be its enveloping action. By arguments similar to those of [4, Lemma 1.13], we can show that, if $L$ is an $\alpha$-prime ideal of $R$, then $L *_{\alpha}^{w} Z$ is a prime ideal of $R *{ }_{\alpha}^{w} Z$. Thus,

$$
\mathrm{Nil}_{*}\left(R *_{\alpha}^{w} \mathbb{Z}\right) \subseteq \operatorname{Nil}_{\alpha}(R) *_{\alpha}^{w} \mathbb{Z},
$$

and, using Proposition 3.4, we have that

$$
\mathrm{Nil}_{*}\left(R *_{\alpha}^{w} \mathbb{Z}\right) \supseteq \mathrm{Nil}_{\alpha}(R) *_{\alpha}^{w} \mathbb{Z} .
$$

This leads to the following result.

Proposition 3.11. Let $\alpha$ be a twisted partial action of $\mathbb{Z}$ on $R$. Then,

$$
\mathrm{Nil}_{*}\left(R *_{\alpha}^{w} \mathbb{Z}\right)=\mathrm{Nil}_{\alpha}(R) *_{\alpha}^{w} \mathbb{Z}
$$

4. Partial crossed products FWPR and AFPR. In this section, we study the necessary and sufficient conditions for the partial crossed products to be fully weakly prime rings. As a consequence of our techniques we obtain the results for the partial crossed products to be FPRs, and we obtain the necessary and sufficient conditions for the partial crossed products to be AFPRs. Moreover, we give some examples to show that our results are not an easy generalization of the global case.

Throughout this section, we assume that the twisted partial action $\alpha$ of $G$ on $R$ has an enveloping action $(T, \beta, u)$, unless otherwise stated.

In [15, Definition 1], a ring $T$ is said to be a $\beta$-FPR if every $\beta$ invariant ideal of $T$ is $\beta$-prime and, in [15, page 86], a $\operatorname{ring} T$ is said to be a $\beta$-AFPR if every proper $\beta$-invariant ideal of $T$ is $\beta$-prime and $T$ is not $\beta$-prime. Now, we need the following definitions.

Definition 4.1. Let $\alpha$ be a twisted partial action of a group $G$ on a ring $R$. 
(i) We say that $R$ is an $\alpha$-FPR if every $\alpha$-invariant ideal of $R$ is $\alpha$-prime.

(ii) We say that $R$ is an $\alpha$-AFPR if every proper $\alpha$-invariant ideal of $R$ is $\alpha$-prime and $R$ is not an $\alpha$-prime ring.

(iii) We say that $R$ is an $\alpha$-FWPR if every proper $\alpha$-invariant ideal of $R$ is weakly $\alpha$-prime.

The proof of the following result is similar to the proof of Proposition 2.19 and will be omitted.

Proposition 4.2. A ring $S$ is an $\alpha-\mathrm{FPR}$ if and only if the set of all $\alpha-$ invariant ideals of $S$ is totally ordered by inclusion and all $\alpha$-invariant ideals of $S$ are idempotent.

We recall that, given an $\alpha$-invariant ideal $I$ of $R$, we have that

$$
I^{*}=\left\{t \in T: \beta_{g}(t) 1_{R} \in I \text { for all } g \in G\right\},
$$

is a $\beta$-invariant ideal of $T$ such that $I^{*} \cap R=I$, see Lemma 2.14. In the next result, we use this fact without further mention.

Proposition 4.3. $R$ is an $\alpha-\mathrm{FWPR}$ if and only if $T$ is a $\beta$-FWPR.

Proof. Let $P$ be a proper $\beta$-invariant ideal of $T$, and assume that $A$ and $B$ are nonzero $\beta$-invariant ideals of $T$ such that $0 \neq A B \subseteq P$. Thus, by similar arguments to those used in the proof of Lemma 2.17, we have

$$
0 \neq(A \cap R)(B \cap R) \subseteq P \cap R,
$$

with $0 \neq A \cap R=A 1_{R}$ and $0 \neq B \cap R=B 1_{R}$. Hence, by assumption, we have that either $A 1_{R} \subseteq P \cap R \subseteq P$ or $B 1_{R} \subseteq P \cap R \subseteq P$. Since $A$, $B$ and $P$ are $\beta$-invariant ideals of $T$, it follows that either $A \beta_{g}\left(1_{R}\right) \subseteq P$ or $B \beta_{g}\left(1_{R}\right) \subseteq P$, for all $g \in G$. So, we have that either $A T \subseteq P$ or $B T \subseteq P$ and, since $T$ is $s$-unital, it follows that either $A \subseteq A T \subseteq P$ or $B \subseteq B T \subseteq P$. Thus, $P$ is weakly $\beta$-prime, and we have that $T$ is a $\beta$-FWPR.

Conversely, let $Q$ be a proper $\alpha$-invariant ideal of $R$, and assume that $I$ and $J$ are $\alpha$-invariant ideals of $R$ such that $0 \neq I, J \subseteq Q$. Since $I^{*} \cap R=I$ and $J^{*} \cap R=J$, we have that $0 \neq I^{*}, J^{*} \subseteq Q^{*}$. By 
assumption, we have that either $I^{*} \subseteq Q^{*}$ or $J^{*} \subseteq Q^{*}$, and it follows that either $I \subseteq Q$ or $J \subseteq Q$. Thus, $Q$ is weakly $\alpha$-prime, and we have that $R$ is an $\alpha$-FWPR.

Using arguments similar to those used in the proof of Proposition 4.3 and using Lemma 2.14, we obtain the following result.

\section{Proposition 4.4.}

(i) $R$ is an $\alpha-\mathrm{FPR}$ if and only if $T$ is a $\beta$-FPR.

(ii) $R$ is an $\alpha$-AFPR if and only if $T$ is a $\beta$-AFPR.

Suppose that $G$ is an infinite cyclic group generated by $\sigma$. In this case, note that $T *_{\beta}^{u} G$ is the twisted skew Laurent polynomial ring $T\langle x ; \sigma, u\rangle$ whose elements are finite sums

$$
\sum_{i=n}^{m} a_{i} x^{i},
$$

where $m, n \in \mathbb{Z}$, with the usual addition of polynomials and multiplication determined by the rule

$$
\left(a_{i} x^{i}\right)\left(a_{j} x^{j}\right)=a_{i} \sigma^{i}\left(a_{j}\right) u_{\sigma^{i}, \sigma^{j}} x^{i+j} .
$$

For each $i, j \in \mathbb{Z}$, we simply denote $u_{\sigma^{i}, \sigma^{j}}$ by $u_{i, j}$. As a subring of $T\langle x ; \sigma, u\rangle$, we have $T[x ; \sigma, u]$, the twisted skew polynomial ring whose elements are the polynomials

$$
\sum_{i=0}^{n} a_{i} x^{i},
$$

with the usual addition and multiplication defined as above, and the leading coefficient of

$$
f=\sum_{i=0}^{n} a_{i} x^{i},
$$

denoted by $\operatorname{lc}(f)=a_{n}$. We define $T_{m}$ as the set of $f \in T[x ; \sigma, u]$ such that $\tau(f) \leq m$, where $\tau(f)$ denotes the degree of the polynomial $f$. Now, let $J$ be a nonzero ideal of $T\langle x ; \sigma, u\rangle$. We define $J \cap T_{m}$ as the 
set $f \in J \cap T\langle x ; \sigma, u\rangle$ such that $\tau(f) \leq m$. Moreover, for an element

$$
\sum_{i=n}^{m} a_{i} x^{i} \in T\langle x ; \sigma, u\rangle,
$$

we define

$$
\sigma^{j}\left(\sum_{i=m}^{n} a_{i} x^{i}\right)=\sum_{i=m}^{n} \sigma^{j}\left(a_{i}\right) x^{i}=x^{j}\left(\sum_{i=m}^{n} a_{i} x^{i}\right) x^{-j} .
$$

An ideal $I$ of $T\langle x ; \sigma, u\rangle$ is said to be $T$-disjoint if $I \cap T=0$. Now, using these facts and with minor adaptations from [19, Lemma 2.11], we have the following result.

Lemma 4.5. Let $I$ be a nonzero $T$-disjoint ideal of $T\langle x ; \sigma, u\rangle$, and let $f \in I$ be a nonzero polynomial of minimal degree $n$ such that $\operatorname{lc}(f)=a$. Suppose that $m \geqslant n$ and $g \in I \cap T_{m}$. If $a_{j} \in T$, and $i_{j}$ is a non-negative integer for each $j \in\{1,2, \ldots, m-n\}$, then there exists $h \in T_{m-n}$ such that

$$
h a_{0} \sigma^{i_{0}}(f)=g \prod_{j=0}^{m-n} \sigma^{-n}\left(a_{m-n-j} \sigma^{i_{m-n-j}}(a)\right),
$$

for all $a_{0} \in T$ and $i_{0} \in \mathbb{Z}$.

Now, we are ready to prove Proposition 4.6 which generalizes [3, Lemma 2.7].

Proposition 4.6. Suppose that $T$ is $\sigma$-prime and $P$ is a nonzero $T$ disjoint ideal of $T\langle x ; \sigma, u\rangle$. Then, $P$ is a prime ideal if and only if $P$ is maximal in the set of $T$-disjoint ideals.

Proof. Suppose that $P$ is a prime ideal of $T\langle x ; \sigma, u\rangle$, and let $I$ be a $T$ disjoint ideal of $T\langle x ; \sigma, u\rangle$ such that $P \subseteq I$. Let $f \in I$ be a polynomial of minimal degree $n$ in $I$ such that $\operatorname{lc}(f)=a$, and let $g \in P$ be a nonzero polynomial of minimal degree $m$ in $P$ such that $\operatorname{lc}(g)=b$. Suppose that $m>n$. For each $g \in P \cap T_{m} \subseteq I \cap T_{m}$, we have, by Lemma 4.5, that there exists $h \in T_{m-n}$ such that

$$
h a_{0} \sigma^{i_{0}}(f)=g \prod_{j=0}^{m-n} \sigma^{-n}\left(a_{m-n-j} \sigma^{i_{m-n-j}}(a)\right),
$$


for all $a_{0} \in T$ and $i_{0} \in \mathbb{Z}$. Since $g \in P$, we obtain that $h T \sigma^{i_{0}}(f) \subseteq P$, for all $i_{0} \in \mathbb{Z}$. Then, for each $t x^{k} \in T\langle x ; \sigma, u\rangle$, we have that $h t \sigma^{k}(f) \sigma^{k}(c) x^{k} \in P$, where $c \in T$ is such that $f c=c$. By the fact that

$$
h t x^{k} f x^{-k} \sigma^{k}(c) x^{k}=h t x^{k} f u_{-k, k},
$$

we have that $h t x^{k} f u_{-k, k} T \subseteq P$. In the proof of [9, Theorem 4.1], we have that $u_{-k, k} T=T u_{-k, k}=T$, and it follows that $h t x^{k} f T \subseteq P$, which implies that $h t x^{k} f \in P$. Consequently, $h T\langle x ; \sigma, u\rangle f \subseteq P$ and, since $P$ is prime, we have that either $h \in P$ or $f \in P$. Thus, either $f=0$ or $h=0$, which contradicts the fact that $h$ and $f$ are nonzero polynomials, and it follows that $m=n$.

Next, let $f \in I$ and $g \in P$ be such that $\tau(f)=n+1$ and $\tau(g)=n$. Then, for

$$
l=a x t x^{i} g c x^{-i}-f \sigma^{-(n+1)}\left(\sigma\left(t \sigma^{i}(b) u_{i, n} u_{n+i,-i}\right) u_{1, n}\right),
$$

where $\operatorname{lc}(g)=b, \operatorname{lc}(f)=a$ and $t \in T$, we easily have that $\tau(l)=n$. Hence, $l \in I \cap T_{n}=P \cap T_{n}$, and it follows that $f T \sigma^{i-n}(b) \subseteq P$, for all $i \in \mathbb{Z}$. Using similar arguments, we obtain that $f \in P$, and consequently, $I \cap T_{n+1}=P \cap T_{n+1}$. Now, proceeding by induction, we have that $I \cap T_{m}=P \cap T_{m}$, for all $m \geqslant 0$. So, $I=P$.

The converse uses arguments similar to those used in [3, Lemma 2.7].

We recall that, given a twisted partial action $\alpha$ of $G$ on a ring $R$, an ideal $J$ of $R *_{\alpha}^{w} G$ is said to be $R$-disjoint if $J \cap R=0$. Lemma 4.7 generalizes [5, Corollary 2.12].

Lemma 4.7. Suppose that $G$ is an infinite cyclic group. If $P_{1}$ and $P_{2}$ are prime ideals of $R *_{\alpha}^{w} G$ such that $P_{1} \cap R=P_{2} \cap R$, then $P_{1}=P_{2}$.

Proof. Since $L=P_{1} \cap R=P_{2} \cap R$ is an $\alpha$-invariant ideal of $R$, we consider the partial crossed product $Z=(R / L) *{ }_{\alpha}^{w} G$. Note that the images of $P_{1}$ and $P_{2}$ in $Z$ are $R / L$-disjoint prime ideals. Thus, we may assume that $P_{1} \cap R=P_{2} \cap R=0$. Since $P_{i}$ is prime, for $i=1,2$, it follows that $R$ is $\alpha$-prime, and we have that $T$ is $\beta$-prime. Again, by the fact that $P_{i} \cap R=0$, we have that the prime ideal $P_{i}^{\prime}$ of $T{ }_{\beta}^{u} G$, such that $P_{i}=P_{i}^{\prime} \cap\left(R *_{\alpha}^{w} G\right)$, satisfies $P_{i}^{\prime} \cap T=0$. Then, by Proposition 4.6, 
we have that $P_{i}^{\prime}$ is maximal in the set of $T$-disjoint ideals of $T *_{\beta}^{u} G$ and, since

$$
P_{i}=P_{i}^{\prime} \cap\left(R *_{\alpha}^{w} G\right),
$$

it is not difficult to see that $P_{i}$ is maximal in the set of the $R$-disjoint ideals of $R *{ }_{\alpha}^{w} G$. Suppose, without loss of generality, that $P_{1} \nsubseteq P_{2}$. Thus, $P_{1} \varsubsetneqq P_{1}+P_{2}$ and, by Proposition 4.6, $P_{1}$ is maximal in the set of $R$-disjoint ideals. It follows that $\left(P_{1}+P_{2}\right) \cap R \neq 0$. Hence, there exists a nonzero element

$$
r=a_{0}+b_{0} \in\left(\left(P_{1}+P_{2}\right) \cap R\right),
$$

such that $a_{0} \in P_{1} \cap R$ and $b_{0} \in P_{2} \cap R$. By the fact that $r \neq 0$, we have that either $a_{0} \neq 0$ or $b_{0} \neq 0$, and we obtain that either $P_{1} \cap R \neq 0$ or $P_{2} \cap R \neq 0$, which is a contradiction. Therefore, $P_{1}=P_{2}$.

In Proposition 4.8, we study partial crossed products by infinite cyclic groups that are FWPR.

Proposition 4.8. Suppose that $G$ is an infinite cyclic group. If $R *_{\alpha}^{w} G$ is an FWPR, then $R$ is an $\alpha-\mathrm{FWPR}$, and there exists a bijective correspondence between the set $\mathcal{L}_{1}$ of the ideals of $R *_{\alpha}^{w} G$ that contains the prime radical of $R *_{\alpha}^{w} G$ and the set $\mathcal{L}_{2}$ of the $\alpha$-invariant ideals of $R$ which contains the $\alpha$-prime radical of $R$.

Proof. Let $A$ be a proper $\alpha$-invariant ideal of $R$, and assume that $I$ and $J$ are $\alpha$-invariant ideals of $R$ such that $0 \neq I J \subseteq A$. By Lemma 2.4 (ii), we have that

$$
0 \neq\left(I *_{\alpha}^{w} G\right)\left(J *_{\alpha}^{w} G\right) \subseteq A *_{\alpha}^{w} G,
$$

and, by assumption, we have that either

$$
I *_{\alpha}^{w} G \subseteq A *_{\alpha}^{w} G \quad \text { or } \quad J *_{\alpha}^{w} G \subseteq A *_{\alpha}^{w} G .
$$

Consequently, either $I \subseteq A$ or $J \subseteq A$.

Note that, by Lemma 3.10, the intersection of all $\alpha$-prime ideals of $R$ is a nilpotent ideal. Now, we define

$$
\Psi: \mathcal{L}_{1} \longrightarrow \mathcal{L}_{2}
$$

by $\Psi(L)=L \cap R$. If $L \in \mathcal{L}_{1}$ and $L$ properly contains $\operatorname{Nil}_{*}\left(R *_{\alpha}^{w} G\right)$, then $L$ is a prime ideal; otherwise, by [14, Proposition 1], we would 
have $L^{2}=0$, and hence, $L=\operatorname{Nil}_{*}\left(R *_{\alpha}^{w} G\right)$. Since $L$ is a prime ideal, by Lemma 2.6 (ii), we have that $L \cap R$ is an $\alpha$-prime ideal of $R$ that contains the $\alpha$-prime radical. By Lemma 4.7 , we easily see that $\Psi$ is injective. We show that $\Psi$ is surjective. In fact, let $K$ be an $\alpha$-invariant ideal of $R$ that properly contains $\operatorname{Nil}_{\alpha}(R)$. If $K$ was nilpotent, then we would have that $K \subseteq \mathrm{Nil}_{\alpha}(R)$, this contradicts the assumption on $K$. Hence, $K^{2}=K$ and, by Lemma $3.2, K$ is $\alpha$-prime. Using the same techniques of [4, Lemma 1.13], we obtain that $K *_{\alpha}^{w} G$ is a prime ideal of $R *{ }_{\alpha}^{w} G$ which contains the prime radical of $R *{ }_{\alpha}^{w} G$.

Lemma 4.9. $R *_{\alpha}^{w} G$ is an FWPR if and only if $T *_{\beta}^{u} G$ is an FWPR.

Proof. Suppose that $R *{ }_{\alpha}^{w} G$ is an FWPR. Let $P^{\prime}$ be a proper ideal of $T *_{\beta}^{u} G$, and assume that $I^{\prime}$ and $J^{\prime}$ are ideals of $T *_{\beta}^{u} G$ such that $0 \neq I^{\prime}$ and $J^{\prime} \subseteq P^{\prime}$. Note that, given an ideal $A^{\prime}$ of $T *_{\beta}^{u} G$, by Lemma 2.10, there exists an ideal $A$ of $R *{ }_{\alpha}^{w} G$ such that $A=A^{\prime} \cap\left(R *{ }_{\alpha}^{w} G\right)$. Moreover, by Corollary 2.11, we have that $A=M A^{\prime} N$ and $A^{\prime}=N A M$. Using these facts and the relations of Lemma 2.7, we obtain that $0 \neq I$, $J \subseteq P$. Since $P$ is weakly prime, we have that either $I \subseteq P$ or $J \subseteq P$. Hence, either $I^{\prime} \subseteq P^{\prime}$ or $J^{\prime} \subseteq P^{\prime}$. Thus, $P^{\prime}$ is weakly prime and so $T *_{\beta}^{u} G$ is an FWPR.

By similar arguments, we obtain the converse.

By Lemma 2.10, there exists a bijective correspondence between the set of the ideals of $R *_{\alpha}^{w} G$ and the set of the ideals of $T *_{\beta}^{u} G$ and, by Lemma 2.12, there exists a bijective correspondence between the set of the prime ideals of $R *_{\alpha}^{w} G$ and the set of the prime ideals of $T *_{\beta}^{u} G$. Thus, we obtain the following result.

\section{Lemma 4.10.}

(i) $R *_{\alpha}^{w} G$ is an $\mathrm{FPR}$ if and only if $T *_{\beta}^{u} G$ is an FPR.

(ii) $R *_{\alpha}^{w} G$ is an AFPR if and only if $T *_{\beta}^{u} G$ is an AFPR.

Using the same arguments as in the proofs of Proposition 4.8 and Corollary 2.15 to prove the injectivity, we obtain the following result. 
Proposition 4.11. Suppose that $G$ is a finite group. If $R *_{\alpha}^{w} G$ is an FWPR, then $R$ is an $\alpha-\mathrm{FWPR}$, and there is a bijective correspondence between the set $\mathcal{L}_{1}$ of the ideals of $R *{ }_{\alpha}^{w} G$ that contains the prime radical of $R *_{\alpha}^{w} G$ and the set $\mathcal{L}_{2}$ of all $\alpha$-invariant ideals of $R$ which contains the $\alpha$-prime radical of $R$.

From now on, we denote the set of the ideals of $R$ by $\mathcal{L}(R)$, the set of the $\alpha$-invariant ideals of $R$ by $\alpha-\mathcal{L}(R)$, and the set of the non-minimal ideals of $R$ by $\overline{\mathcal{L}(R)}$.

Theorem 4.12. Suppose that $G$ is any group. If $R$ is an $\alpha-\mathrm{FWPR}$ and the map,

$$
\varphi: \mathcal{L}\left(R *_{\alpha}^{w} G\right) \longrightarrow \alpha-\mathcal{L}(R)
$$

defined by $\varphi(J)=J \cap R$, is bijective, then $R *_{\alpha}^{w} G$ is an FWPR.

Proof. Note that, by injectivity of $\varphi$, we have $(A \cap R) *_{\alpha}^{w} G=A$, for any ideal $A$ of the $R *_{\alpha}^{w} G$. Let $P$ be a proper ideal of $R *_{\alpha}^{w} G$, and assume that $I$ and $J$ are ideals of $R *{ }_{\alpha}^{w} G$ such that $0 \neq I, J \subseteq P$. Since

$$
I J=\left[(I \cap R) *_{\alpha}^{w} G\right]\left[(J \cap R) *_{\alpha}^{w} G\right] \subseteq(I \cap R)(J \cap R) *_{\alpha}^{w} G,
$$

it follows that $(I \cap R)(J \cap R) \neq 0$. Then $0 \neq(I \cap R)(J \cap R) \subseteq(P \cap R)$ and, by the fact that $R$ is an $\alpha$-FWPR, we have that either $I \cap R \subseteq P \cap R$ or $J \cap R \subseteq P \cap R$. Hence, either

$$
I=(I \cap R) *_{\alpha}^{w} G \subseteq(P \cap R) *_{\alpha}^{w} G=P,
$$

or

$$
J=(J \cap R) *_{\alpha}^{w} G \subseteq(P \cap R) *_{\alpha}^{w} G=P .
$$

Thus, $P$ is weakly prime and so $R *_{\alpha}^{w} G$ is an FWPR.

The next proposition generalizes [15, Theorem 2].

Proposition 4.13. Suppose that $G$ is a finite group. Then $R *_{\alpha}^{w} G$ is an FPR if and only if $R$ is an $\alpha-\mathrm{FPR}$, and the map $\phi: \mathcal{L}\left(R *_{\alpha}^{w} G\right) \rightarrow$ $\alpha-\mathcal{L}(R)$, defined by $\phi(I)=I \cap R$, is bijective.

Proof. Suppose that $R *{ }_{\alpha}^{w} G$ is an FPR. Let $A$ be an $\alpha$-invariant ideal of $R$, and assume that $I$ and $J$ are $\alpha$-invariant ideals of $R$ such that 
$I J \subseteq A$. Then

$$
\left(I *_{\alpha}^{w} G\right)\left(J *_{\alpha}^{w} G\right) \subseteq A *_{\alpha}^{w} G,
$$

and, by assumption, we have that either $I *_{\alpha}^{w} G \subseteq A *_{\alpha}^{w} G$ or $J *_{\alpha}^{w} G \subseteq$ $A *_{\alpha}^{w} G$. Consequently, either $I \subseteq A$ or $J \subseteq A$. Thus, $A$ is $\alpha$-prime and so $R$ is an $\alpha$-FPR. By Lemma 2.4 (ii), clearly $\phi$ is surjective. Moreover, $\phi$ is injective because, if $I$ and $J$ are ideals of $R *_{\alpha}^{w} G$ such that $I \cap R=J \cap R$, then by Lemma 2.15, we have that $I=J$.

By similar arguments to those of Theorem 4.12, we obtain the converse.

Lemma 4.14. Let $\alpha$ be a twisted partial action of a finite group $G$ on $R$. Suppose that $R *_{\alpha}^{w} G$ is an AFPR whose set of ideals is not totally ordered by inclusion, and let $P_{1}$ and $P_{2}$ be the minimal ideals of $R *_{\alpha}^{w} G$. Then, $P_{1} \cap R=0$ if and only if $P_{2} \cap R=0$.

Proof. If $P_{1} \cap R=0$, then by Lemma 2.6 (i) we have that $0=P_{1} \cap R$ is an $\alpha$-prime ideal of $R$. Thus, $R$ is $\alpha$-prime. Since $P_{2}$ is minimal, then by Corollary 2.16, we have that $P_{2} \cap R=0$. We obtain the converse analogously.

Lemma 4.15. Suppose that $\alpha$ is a twisted partial action of a group $G$ on a ring $R$ which is an $\mathrm{AFPR}$. If the map

$$
\phi: \mathcal{L}\left(R *_{\alpha}^{w} G\right) \longrightarrow \mathcal{L}(R),
$$

defined by $P \mapsto P \cap R$, is bijective, then all proper ideals of $R *_{\alpha}^{w} G$ are prime.

Proof. By arguments similar to those of Theorem 4.12, we obtain the result.

In the next result, we study the sufficient conditions for the partial crossed product to be an AFPR and it partially generalizes [15, Theorems 4.5]. Moreover, we do not know if the converse of the next result is true.

Theorem 4.16. Suppose that $\alpha$ is a twisted partial action of a finite group $G$ on $R$ which is an AFPR. If one of the following conditions is satisfied, then $R *_{\alpha}^{w} G$ is an AFPR. 
(i) The map $\phi_{1}: \mathcal{L}\left(R *_{\alpha}^{w} G\right) \rightarrow \mathcal{L}(R)$, defined by $P \mapsto P \cap R$, is bijective;

(ii) (a) $R *_{\alpha}^{w} G$ has exactly two minimal ideals, $P_{1}$ and $P_{2}$, which are prime;

(b) the map $\phi_{2}: \overline{\mathcal{L}\left(R *_{\alpha}^{w} G\right)} \rightarrow \overline{\mathcal{L}(R)}$, defined by $P \mapsto P \cap R$, is bijective.

(iii) (a) $R *_{\alpha}^{w} G$ has only one minimal ideal $P_{0}$ which is prime and nilpotent;

(b) the map $\phi_{3}: \overline{\mathcal{L}\left(R *_{\alpha}^{w} G\right)} \rightarrow \overline{\mathcal{L}(R)}$, defined by $P \mapsto P \cap R$, is bijective.

Proof. Suppose that (i) holds. We have two cases to consider.

Case 1. $\mathcal{L}(R)$ is totally ordered by inclusion. By Lemma 2.21, $R$ has a unique minimal nilpotent ideal $Q_{0}$, and we easily obtain that $P_{0}=Q_{0} *_{\alpha}^{w} G$ is a nilpotent minimal ideal of $R *_{\alpha}^{w} G$. Hence, $R *_{\alpha}^{w} G$ is not prime. Moreover, by Lemma 4.15, all proper ideals of $R *{ }_{\alpha}^{w} G$ are prime. So, $R *_{\alpha}^{w} G$ is an AFPR.

Case 2. $\mathcal{L}(R)$ is not totally ordered by inclusion. By Lemma 2.20, $R$ has two minimal ideals, $Q_{1}$ and $Q_{2}$. Since $\phi$ is bijective, there exist nonzero ideals $P_{1}$ and $P_{2}$ of $R *_{\alpha}^{w} G$ such that $P_{1} \cap R=Q_{1}$ and $P_{2} \cap R=Q_{2}$. It is not difficult to show that $P_{1}$ and $P_{2}$ are minimal prime ideals of $R * *_{\alpha}^{w} G$. Thus, $P_{1} P_{2}=0$, and it follows that $R *_{\alpha}^{w} G$ is not prime. Moreover, by Lemma 4.15, all proper ideals of $R *_{\alpha}^{w} G$ are prime. So, $R *_{\alpha}^{w} G$ is an AFPR.

Suppose that (ii) holds. Let $P$ be a proper ideal of $R *_{\alpha}^{w} G$. If $P$ is minimal, then $P$ is prime, by (a). If $P$ is not minimal, then $P \in \overline{\mathcal{L}\left(R *_{\alpha}^{w} G\right)}$ and, by the fact that $\phi_{2}$ is bijective, we have, by arguments similar to those used in the proof of Theorem 4.12, that $P$ is prime. So, any proper ideal of $R *_{\alpha}^{w} G$ is prime. Moreover, since $P_{1}$ and $P_{2}$ are minimal ideals of $R *_{\alpha}^{w} G$, we have that $P_{1} P_{2}=0$, and it follows that $R *_{\alpha}^{w} G$ is not prime. Hence, $R *_{\alpha}^{w} G$ is an AFPR.

Suppose that (iii) holds. By analogous methods to those used in the proof of (ii), we obtain that any proper ideal of $R *_{\alpha}^{w} G$ is prime. Since, by (a), the minimal ideal $P_{0}$ is nilpotent, it follows that $R *_{\alpha}^{w} G$ is not prime. So, $R *_{\alpha}^{w} G$ is an AFPR. 
Theorem 4.17. [15, Theorems 6, 7]. Let $\alpha$ be a twisted partial action of a finite group $G$ on $R$. Then, $R *{ }_{\alpha}^{w} G$ is an AFPR if and only if either:

(i) (a) $R$ is an $\alpha$-AFPR;

(b) the map $\phi_{1}: \mathcal{L}\left(R *_{\alpha}^{w} G\right) \rightarrow \alpha-\mathcal{L}(R)$, defined by $P \mapsto P \cap R$, is bijective; or,

(ii) (a) $R$ is an $\alpha$-FPR;

(b) the minimal ideals of $R *{ }_{\alpha}^{w} G$ are prime;

(c) the map $\phi_{2}: \overline{\mathcal{L}\left(R *_{\alpha}^{w} G\right)} \rightarrow \alpha-\mathcal{L}(R)$, defined by $P \mapsto P \cap R$, is bijective.

Proof. Suppose that $R *_{\alpha}^{w} G$ is an AFPR. We have two cases to consider.

Case 1. $\mathcal{L}\left(R *_{\alpha}^{w} G\right)$ is not totally ordered by inclusion. By Lemma 2.20 (i), $R *_{\alpha}^{w} G$ has two minimal ideals, $P_{1}$ and $P_{2}$, which are prime because $R *{ }_{\alpha}^{w} G$ is an AFPR. Now, we have the following subcases.

Subcase 1.1. $P_{1} \cap R \neq 0$. By methods analogous to those used in the proof of Proposition 4.13, we have that all nonzero $\alpha$-invariant ideals of $R$ are $\alpha$-prime. Since $P_{1} \cap R \neq 0$, by Lemma 4.14, we have that $P_{2} \cap R \neq 0$. Thus, $\left(P_{1} \cap R\right)\left(P_{2} \cap R\right) \subseteq P_{1} P_{2}=0$, and consequently, $R$ is not $\alpha$-prime. Hence, $R$ is an $\alpha$-AFPR.

Using Corollary 2.15 and Lemma 2.4 (ii), we easily obtain that $\phi_{1}$ is bijective.

Subcase 1.2. $P_{1} \cap R=0$. By the same arguments as those of subcase 1.1, we have that all nonzero $\alpha$-invariant ideals of $R$ are $\alpha$ prime. Since, by Lemma 2.6 (i), $P_{1} \cap R=0$, then $R$ is $\alpha$-prime, and it follows that $R$ is an $\alpha$-FPR.

Note that, for each $P \in \overline{\mathcal{L}\left(R *_{\alpha}^{w} G\right)} \backslash\{0\}$, we have that $\phi_{2}(P)=$ $P \cap R \neq 0$; otherwise, $P$ would be minimal by Corollary 2.16 since $R$ is $\alpha$-prime. By the facts that $\operatorname{Nil}_{\alpha}(R)=0$ and $\operatorname{Nil}_{*}\left(R *_{\alpha}^{w} G\right)=$ $P_{1} \cap P_{2}=0$, we have, by similar arguments to those used in the proof of Proposition 4.11, that $\phi_{2}$ is bijective.

Case 2. $\mathcal{L}\left(R *_{\alpha}^{w} G\right)$ is totally ordered by inclusion. By Lemma 2.21, $R *_{\alpha}^{w} G$ has a unique minimal nilpotent ideal $P_{0}$ which is prime because $R *{ }_{\alpha}^{w} G$ is an AFPR. Now, we have the following subcases. 
Subcase 2.1. $P_{0} \cap R \neq 0$. By arguments analogous to those of Proposition 4.13, we have that all nonzero $\alpha$-invariant ideals of $R$ are $\alpha$-prime. By the fact that $\left(P_{0}\right)^{2}=0$ we have that $\left(P_{0} \cap R\right)\left(P_{0} \cap R\right) \subseteq$ $\left(P_{0}\right)^{2}=0$, with $P_{0} \cap R \neq 0$. Hence, $R$ is not $\alpha$-prime, and it follows that $R$ is an $\alpha$-AFPR. Moreover, analogously to subcase 1.1, we obtain that $\phi_{1}$ is bijective.

Subcase 2.2. $\quad P_{0} \cap R=0$. By similar arguments to those of subcase 1.2 , we obtain that $R$ is an $\alpha$-FPR, and $\phi_{2}$ is bijective.

Conversely, suppose that Theorem 4.17 (i) holds. Since $\phi_{1}$ is bijective, then by analogous techniques to Proposition 4.13, we show that all proper ideals of $R *_{\alpha}^{w} G$ are prime. Since $R$ is an $\alpha$-AFPR, there exist nonzero $\alpha$-invariant ideals $A$ and $B$ of $R$ such that $A B=0$. Hence, $\left(A *_{\alpha}^{w} G\right)\left(B *_{\alpha}^{w} G\right)=0$, and we have that $R *_{\alpha}^{w} G$ is not prime. So, $R *_{\alpha}^{w} G$ is an AFPR.

Suppose that Theorem 4.17 (ii) holds. Let $P$ be a proper ideal of $R * *_{\alpha}^{w} G$. If $P$ is minimal then, by Theorem 4.17 (ii) (b), $P$ is prime. If $P$ is not minimal, let $I$ and $J$ be ideals of $R *_{\alpha}^{w} G$ such that $I, J \subseteq P$. Then,

$$
(I \cap R)(J \cap R) \subseteq P \cap R .
$$

Since $R$ is an $\alpha$-FPR, we have that $P \cap R$ is $\alpha$-prime, and it follows that either $I \cap R \subseteq P \cap R$ or $J \cap R \subseteq P \cap R$. By the fact that $\phi_{2}$ is bijective, we have that

$$
\begin{aligned}
& I=(I \cap R) *_{\alpha}^{w} G, \\
& J=(J \cap R) *_{\alpha}^{w} G,
\end{aligned}
$$

and

$$
P=(P \cap R) *_{\alpha}^{w} G
$$

Consequently, either

$$
I=(I \cap R) *_{\alpha}^{w} G \subseteq(P \cap R) *_{\alpha}^{w} G=P
$$

or

$$
J=(J \cap R) *_{\alpha}^{w} G \subseteq(P \cap R) *_{\alpha}^{w} G=P .
$$

Hence, all proper ideals of $R *_{\alpha}^{w} G$ are prime. Now, if $\mathcal{L}\left(R *_{\alpha}^{w} G\right)$ is not totally ordered by inclusion, then there exist nonzero ideals $I$ and $J$ of $R *_{\alpha}^{w} G$ such that $I \nsubseteq J$ and $J \nsubseteq I$. Note that $I \cap J=0$; otherwise, 
$I \cap J$ would be prime, and we would obtain that $I \subseteq I \cap J \subseteq J$ or $J \subseteq I \cap J \subseteq I$, which is a contradiction. Consequently, $I J \subseteq I \cap J=0$, and we have that $R *_{\alpha}^{w} G$ is not a prime ring. Now, suppose that $\mathcal{L}\left(R *_{\alpha}^{w} G\right)$ is totally ordered by inclusion. In this case, $R *_{\alpha}^{w} G$ has a minimal ideal $P_{0}$. Note that $P_{0} \cap R$ is an $\alpha$-invariant ideal of $R$. Thus, there exists an ideal $P \in \overline{\mathcal{L}\left(R *_{\alpha}^{w} G\right)}$ such that $P \cap R=P_{0} \cap R$. So, $P=0$; otherwise, we would have that $P$ is prime and, by Corollary 2.15 , it follows that $P=P_{0}$. Thus, $P_{0} \cap R=0$. We clearly have that $R *_{\alpha}^{w} G$ is not a prime ring because, if 0 were prime, then, by Corollary 2.15, we would have that $P_{0}=0$, which is a contradiction. So, $R *_{\alpha}^{w} G$ is an AFPR.

It is natural to ask if $R$ is either FWPR, AFPR, FPR, or if the set of ideals is totally ordered by inclusion, then would $T$ either be FWPR, AFPR, FPR, or would the set of ideals of $T$ be totally ordered by inclusion? The examples below show that this is not the case and that our results are not an easy generalization of the global case.

\section{Example 4.18.}

(i) Let $K$ be a field, and let $\left\{e_{i}: i \in \mathbb{Z}\right\}$ be a set of orthogonal central idempotents and $T=\oplus_{i \in \mathbb{Z}} K e_{i}$. We define a global action of the infinite cyclic group $G$ generated by $\sigma$ on $T$ by $\sigma\left(e_{i}\right)=e_{i+1}$, for all $i \in \mathbb{Z}$. If $R=K e_{0}$, then we clearly have a partial action of the group $G$ on $R$. Note that $R$ is an FPR, but $T$ is not an FPR. Moreover, all ideals of $R$ are totally ordered by inclusion, but the set of the ideals of $T$ is not totally ordered by inclusion. In turn, if $R=K e_{0} \oplus K e_{1}$, then $R$ is an AFPR, but $T$ is not an AFPR.

(ii) Let $K$ be a field, let $\left\{e_{1}, e_{2}, e_{3}, e_{4}\right\}$ be a set of orthogonal central idempotents and let $T=\oplus_{i=1}^{4} K e_{i}$. We define a global action of the finite cyclic group of order 4 generated by $\sigma$ on $T$ by $\sigma\left(e_{1}\right)=e_{2}$, $\sigma\left(e_{2}\right)=e_{3}, \sigma\left(e_{3}\right)=e_{4}$ and $\sigma\left(e_{4}\right)=e_{1}$. If $R=K e_{1}$, then we clearly have a partial action of $G$ on $R$. Note that $R$ is an FPR, but $T$ is not an FPR. Moreover, all ideals of $\mathrm{R}$ are totally ordered by inclusion, but the set of ideals of $\mathrm{T}$ is not totally ordered by inclusion.

(iii) Let $T$ and $\sigma$ be as in (ii). If $R=K e_{1} \oplus K e_{2}$, then we clearly have a partial action of $G$ on $R$. Note that $R$ is an AFPR, but $T$ is not an AFPR. Moreover, note that $R$ is an FWPR, but $T$ is not an 
FWPR because we let $I=K e_{1} \oplus K e_{3}$ and $J=K e_{1} \oplus K e_{2}$. Then $0 \neq I, J \subseteq K e_{1} \oplus K e_{4}$, but $I \nsubseteq K e_{1} \oplus K e_{4}$ and $J \nsubseteq K e_{1} \oplus K e_{4}$.

Remark 4.19. Let $\beta$ be a twisted global action of a group $G$ on a ring $T$. If the set of all the ideals of $T$ are totally ordered by inclusion, then all the ideals of $T$ are $\beta$-invariant. If it is possible to generalize this fact to twisted partial actions, then it is possible to prove the converse of Theorem 4.16. However, until now, we could not prove this fact, nor provide a counterexample.

\section{REFERENCES}

1. W.D. Blair and H. Tsutsui, Fully prime rings, Comm. Alg. 22 (1994), 53885400.

2. P. Carvalho, W. Cortes and M. Ferrero, Partial skew group rings over polycyclic by finite groups, Alg. Rep. Th. 14 (2011), 449-462.

3. E. Cisneros, M. Ferrero and M.I. Conzáles, Prime ideals of skew polynomial rings and skew Laurent polynomial rings, Math. J. Okayama Univ. 32 (2007), 6172 .

4. W. Cortes, Partial skew polynomial rings and Jacobson rings, Comm. Alg. 38 (2010), 1526-1548.

5. W. Cortes and M. Ferrero, Partial skew polynomial rings: Prime and maximal ideals, Comm. Alg. 35 (2007), 1183-1199.

6. M. Dokuchaev, A. del Rio and J.J. Simón, Globalizations of partial actions on nonunital rings, Proc. Amer. Math. Soc. 135 (2007), 343-352.

7. M. Dokuchaev and R. Exel, Associativity of crossed products by partial actions, enveloping actions and partial representations, Trans. Amer. Math. Soc. 357 (2005), 1931-1952.

8. M. Dokuchaev, R. Exel and J.J. Simón, Crossed products by twisted and graded algebras, J. Alg. 320 (2008), 3278-3310.

9. Globalization of twisted partial actions, Trans. Amer. Math. Soc. 362 (2010), 4137-4160.

10. M. Dokuchaev, M. Ferrero and A. Paques, Partial actions and Galois theory, J. Pure Appl. Alg. 208 (2007), 77-87.

11. R. Exel, Twisted partial actions: A classification of regular $C^{*}$-algebraic bundles, Proc. Lond. Math. Soc. 74 (1997), 417-443.

12. R. Exel, M. Laca and J. Quigg, Partial dynamical system and $C^{*}$-algebras generated by partial isometries, J. Oper. Theor. 47 (2002), 169-186.

13. M. Ferrero and J. Lazzarin, Partial actions and partial skew group rings, J. Alg. 139 (2008), 5247-5264. 
14. Y. Hirano, E. Poon and H. Tsutsui, On rings in which every ideal is weakly prime, Bull. Korean Math. Soc. 47 (2010), 1077-1087.

15. L. Huang and $\mathrm{Z}$. Yi, Crossed products and full prime rings, in Advances in ring theory, Proc. 4th China-Japan-Korea Intl. Conf., 2005.

16. T.Y. Lam, A first course in noncommutative rings, Springer-Verlag, New York, 2001.

17. K. McClanahan, K-theory for partial crossed products by discrete groups, J. Funct. Anal. 130 (1995), 77-117.

18. D.S. Passman, Infinite crossed products, Academic Press, Cambridge, MA, 1980.

19. K.R. Pearson, W. Stephenson and J.F. Watters, Skew polynomials and Jacobson rings, Proc. Lond. Math. Soc. 42 (1981), 559-576.

20. J.C. Quigg and I. Raeburn, Characterizations of crossed products by partial actions, J. Oper. Th. 37 (1997), 311-340.

21. H. Tsutsui, Fully prime rings II, Comm. Alg. 24 (1996), 2981-2989.

Instituto de Matemática, Universidade Federal do Rio Grande do Sul, Porto Alegre, RS, 91509-900 Brazil

Email address: cortes@mat.ufrgs.br

Departamento de Matemática, Universidade Estadual do Centro-Oeste, Guarapuava, PR, 85055-250 BrazIL

Email address: marlonsoares@unicentro.br 